Medical treatment is rarely recommended. The aim of our study is to evaluate follow-up and response to medical treatment in patients affected by hydatid cystis (HC).

Methods We conducted a retrospective study about all cases of $\mathrm{HC}$ treated by albendazole in paediatrics department of Hedi Chaker University Hospital in Sfax over a period of 10 years (2004-2013).

Results We report 6 cases of HC treated medically. Ages ranged between 2 and 8 years. Diagnosis was based on laboratory and imaging studies. Hydatid cystis had multiple localizations in all of cases: multiple hepatic HC (1 case), bilateral pulmonary HC (1 case), hepatic and pulmonary HC (1 case), hepatic and splenic $\mathrm{HC}$, hepatic, splenic and renal HC and hepatic, pulmonary, cardiac and pancreatic HC. All patients were treated with Albendazole $(10 \mathrm{mg} / \mathrm{kg} / \mathrm{day}$ during 6 to 24 months). Surgical treatment was associated in 4 cases. The outcome was favourable in all cases.

Conclusions Surgery remains the ideal treatment for hydatid cysts. However, medical treatment with albendazole can be efficient and can, in some circumstances, replace surgery especially in case of multiple locations.

\section{PO-0250 CEREBROSPINAL FLUID CYTOKINE IN CENTRAL NERVOUS SYSTEM INVOLVEMENT ASSOCIATED WITH ROTAVIRUS INFECTION}

S Suzuki, Y Kashiwagi, H Kawashima. Pediatrics, Tokyo Medical University, Tokyo, Japan

10.1136/archdischild-2014-307384.901

Introduction Rotavirus is the most common cause of severe gastroenteritis in young children, but the pathogenesis of this disease and immunity to it are not completely understood. Less well recognised is rotavirus-induced central nervous system (CNS) involvement, which has been associated with seizure, encephalopathy and death.

Subjects and method We enrolled 20 children hospitalised in Tokyo Medical University (Tokyo, Japan) between 1999 and 2009. They had been given diagnosis of rotavirus- induced CNS involvement. Subjects were classified into two groups of patients with encephalopathy following prolonged seizure (encephalopathy group) and afebrile, and those with a brief cluster of seizures without encephalopathy (cluster group). We assayed 10 cytokines (IL-1 $\beta$, IL-6, IL-8, IL-10, IL-12, IL-17, TNF- $\alpha$, IFN- $\gamma$, MCP-1 and G-CSF) in the CSF obtained from patients.

Result IL- $1 \beta$ and IL- 6 increased in the group of children with encephalopathy compared with those in the cluster group. On the other hand, IL-17 levels of the cluster group were significantly higher than those of the encephalopathy group.

Discussion High cytokine levels in CSF may induce neurological disorders and may be related to the severity of disorders. This is, to the best of our knowledge, the first report about several cytokines in CSF obtained from patients with CNS involvement associated with rotavirus infection. Further study is necessary to determine whether CSF cytokine have an important role in rotavirus infection-induced CNS involvement.

\section{PO-0251 TUBERCULOSIS IN CHILDREN: A STUDY OF 32 CASES}

M Tfifha, D Dhahri, S Mabrouk, H Ajmi, J Chemli, S Hassayoun, N Zouari, S Abroug. Pediatrics, University Hospital Sahloul, Sousse, Tunisia

10.1136/archdischild-2014-307384.902
Introduction Tuberculosis (TBC) continues to cause an unacceptably high toll of disease and death among children worldwide, particularly in the developing countries.

Objective Study the epidemiological and clinical aspects of TBC in Tunisian children and clarify its treatment.

Population and methods A descriptive study was carried out, included all cases of TBC diagnosed in Sahloul paediatric unit hospital between 2004 and 2013. Epidemiological, clinical and microbiological data were recorded. Therapeutic and evolutionary aspects were clarified.

Results We reviewed 32 cases (16 boys and 16 girls), aged from 6 months to 15 years. All patients were vaccinated against tuberculosis. No case of malnutrition none immune deficiency had been recorded until the first hospitalisation. The diagnosis of TBC was based on clinical features, tuberculin skin test, chest radiography and the histological examination of lymph node, peritoneal and pleural biopsy. Diagnoses were identified: Lymph node tuberculosis (11 cases), pulmonary tuberculosis (4 cases), peritoneal tuberculosis (4 cases), bone tuberculosis (4 cases), tuberculous meningitis ( 2 cases), cutaneous tuberculosis (1 case), renal tuberculosis (1 case), tuberculosis in hematopoietic stem cell (1 case), pleuro-pericarditis tuberculosis (1 case), and the disease has affected several organs (disseminated) in 3 cases. Two cases of congenital immunodeficiency syndrome were identified. All our patients were treated with an association of Isoniazid, Rifampicin, Pyrazinamide and Ethambutol for 2 months, and then they received Isoniazid and Rifampicin for a period ranging from 2 to 16 months. 27 children were cured (24 without sequelae and 3 with sequelae). Two children died.

Conclusion Tuberculosis in children presents particularly difficult challenges, but advances in paediatric tuberculosis research could provide wider insights and opportunities for tuberculosis control especially in developing countries.

\section{PO-0252 WITHDRAWN}

\section{PO-0253 USE OF INFECTION MARKERS FOR DIAGNOSTIC AND MANAGEMENT OF SUSPECTED NEONATAL EARLY ONSET NEONATAL SEPSIS: AN INTERNATIONAL SURVEY}

${ }^{1}$ W van Herk, ${ }^{2}$ S El Helou, ${ }^{3}$ J Janota, ${ }^{4} \mathrm{C}$ Hagmann, ${ }^{5} \mathrm{C}$ Klingenberg, ${ }^{6} \mathrm{E}$ Staub, ${ }^{7} \mathrm{E}$ Giannoni, ${ }^{8} \mathrm{~S}$ Pilgrim, ${ }^{9} \mathrm{~L}$ Schlapbach, ${ }^{10 \mathrm{P}}$ Tissieres, ${ }^{1} \mathrm{~A}$ van Rossum, ${ }^{11} \mathrm{M}$ Stocker. ${ }^{1}$ Pediatric Infectious Diseases and Immunology, Erasmus MC Sophia Children's Hospital, Rotterdam, Netherlands; ' Division of Neonatology in the Department of Pediatrics, McMaster University, Hamilton, Canada; ${ }^{3}$ Department of Neonatology, Thomayer Hospital, Prague, Czech Republic; ${ }^{4}$ Division of Neonatology, University Hospital Zurich, Zurich, Switzerland; ${ }^{5}$ Department of Pediatrics, University Hospital of North Norway, Tromsø, Norway; ${ }^{6}$ Pediatric Intensive Care Unit, Children's Hospital Westmaed, Sydney, Australia; ${ }^{7}$ Division of Neonatology, University Hospital of Lausanne, Lausanne, Switzerland; ${ }^{8}$ Division of Neonatology, University Children's Hospital Berne, Berne, Switzerland; ${ }^{9}$ Pediatric Critical Care Research Group, Mater Children's Hospital University of Queensland, Brisbane, Australia; ${ }^{10}$ Department of Pediatric and Neonatal Intensive Care, 10) Hôpitaux Universitaires Paris-Sud AP-HPLe Kremlin-Bicêtre, Paris, France; "11 Division of Neonatology, Children's Hospital Lucerne, Lucerne, Switzerland

\subsection{6/archdischild-2014-307384.903}

Background Early diagnosis and treatment of neonatal earlyonset sepsis (EOS) is essential to prevent severe and life threatening complications. Diagnosis is difficult because of the variable and nonspecific clinical presentation.

Aim To determine the use of laboratory investigations for the decision to start/stop antibiotic therapy and to determine the duration of empiric therapy in infection risk adjusted scenarios. 
Methods Web-based questionnaire (Survey monkey ${ }^{\circledR}$ ), developed by the NEonatal Sepsis Trial NETwork (http://www.nest-net.org), was sent to neonatologists worldwide. Questions regarding management $(n=7)$ were introduced by scenarios levelled to low-, medium- and high risk for neonatal EOS. Demographic questions $(n=4)$ are based on competency, caseload, experience of fatal cases (deaths) and country of origin.

Results 439 Neonatologist from 10 countries participated. Laboratory investigations are used in $31 \%$ to start, and in $72 \%$ to stop antibiotic treatment. The decision regarding stop of antibiotic therapy is mainly dependent on conventional laboratory investigations. Only a minority uses newer infection markers as procalcitonin (17\%) or interleukins (9\%). There is a high variance in when to start and when to stop antibiotic therapy with a national distribution. Variance is lower within one country compared to the variance in all participating countries. There is no dependency on other demographic variables.

Conclusions There is a high variance in the management of neonatal EOS. Discontinuation has a high dependency on laboratory infection markers. Clinical research should focus on safety and predictive values of (new) infection markers to support the decision to stop antibiotic therapy early and prevent possibly unnecessary antibiotic treatment.

\section{PO-0254 TUBERCULOSIS OUTBREAK IN A DAYCARE CENTRE}

${ }^{1}$ A Vásquez, ${ }^{1} \mathrm{O}$ Calavia, ${ }^{1} \mathrm{M}$ Alvarez, ${ }^{2} \mathrm{~J}$ Ventosa, 'L Fernandez, ${ }^{1} \mathrm{R}$ Closa. ${ }^{1}$ Pediatrics, Hospital Joan XXIII, Tarragona, Spain; ${ }^{2}$ Pulmonology, Hospital Joan XXIII, Tarragona, Spain

\subsection{6/archdischild-2014-307384.904}

Objetives The control of tuberculosis (TB) transmission and prevention of outbreaks requires appropriate studies for the contacts. We're going to present an outbreak of tuberculosis in a daycare centre.

Methods A case of pulmonary TB were reported in a 3 years old boy from a daycare, admitted with pneumonia without response to conventional antibiotic therapy, tuberculin test (PPD):5 mm, positive quantiferon. Study of family contacts was negative.

After that pulmonary $\mathrm{TB}$ was confirmed in a caregiver from the daycare, she was considered baciliferus and also the index case.

Results Contact study was performed in 90 persons exposed. $85 \%(77 / 90)$ contacts from the daycare (67 children under 3 years and 10 adults). 15 people had positive tuberculin, 8 contacts from the daycare ( 7 children and 1 caregiver). 5 patients of the group mentioned before were considered latent tuberculosis infection, receiving secondary chemoprophylaxis and 3 were diagnosed with tuberculosis disease, being treated with satisfactory outcome in all cases.

The index case had over a month off work so that children with negative tuberculin was repeated at 5 weeks being negative in all cases.

Conclusions TB in children can be confused with other typical lung infections, however, must be ruled out if it has a subacute respiratory symptoms and poor response to conventional treatment. It's really important the rapid detection of contacts incase of adults with TB disease, especially if those work with susceptible people like children. The PPD is still an easy and simple tool for unvaccinated contacts diagnostic.

\section{PO-0255 FEATURES OF HOSPITALISED CHILDREN SUSPECTED INFECTION WITH INFLUENZA A H1N1 SUBTYPE IN PAEDIATRICS OF TEACHING HOSPITAL ALCIDES CARNEIRO (HEAC), PETRÓPOLIS, RJ, BRAZIL}

${ }^{1} \mathrm{E}$ Veiga, ${ }^{2} \mathrm{~A}$ Veiga, ${ }^{3} \mathrm{C}$ Tammela, ${ }^{3} \mathrm{~F}$ Ferreira, ${ }^{3} \mathrm{~J}$ Souza, ${ }^{4} \mathrm{~S}$ Nogueira, ${ }^{4} \mathrm{~S}$ Cordeiro, ${ }^{4} \mathrm{~F}$ Moliterno, ${ }^{4} \mathrm{~N}$ Moliterno. ${ }^{1}$ Pediatria, Faculdade Medicina de Petrópolis, Petrópolis, Brazil; ${ }^{2}$ Pediatria, Faculdade Medicina de Petrópolis, Petrópolis, Brazil; ${ }^{3}$ Graduação Em Medicina, Faculdade Medicina de Petrópolis, Petrópolis, Brazil; ${ }^{4}$ Pediatria, Faculdade Medicina de Petrópolis, Petrópolis, Brazil

\subsection{6/archdischild-2014-307384.905}

Introduction Influenza A virus subtype H1N1 has been identified as an important pathogen in childhood morbidity. Taking into account the post-pandemic situation in 2009, knowledge of the epidemiology of influenza in the paediatric population is essential for better targeting of strategies for clinical management, therapeutic and epidemiological control.

Objective To describe the profile of children hospitalised with suspected H1N1 infection in the paediatric ward of HEAC, during the year 2013.

Methods Cross-sectional, retrospective study, simple analytical paediatric hospitalizations for suspected H1N1.

Results and discussion During the study period 1039 paediatric hospitalizations were reported, including 349 (33\%) for respiratory diseases. Of these, 21 (6\%) used Oseltamivir Phosphate for suspected $\mathrm{H} 1 \mathrm{~N} 1$ from June, focusing more in winter and spring. The age ranged between $2 \mathrm{~m}$ and 11th with an average of 3.16 years. Male predominance $76.19 \%$. The mean hospital stay was 10,33 days. Comorbidities such as encephalopathy, heart diseases, obesity in 17 (80.9\%), and age <2 years in 10 (47.6\%). Were referred to the Intensive Therapy $23.8 \%$. Specific oropharyngeal swabs collected 9 (42.85\%), 3 of these $(14.28 \%)$ confirmed the diagnosis. All were classified as Severe Acute Respiratory Syndrome, as the Protocol of the Ministry of Health Only $6(28.5 \%)$ began specific therapy within the first $48 \mathrm{~h}$, as recommended as an effective treatment.

Conclusion Influenza will remain a challenge for medicine over the years, requiring proactive management. The epidemiological report suspected front, start the appropriate therapy, the specific diagnostic screening and recognition of clinical severity, can contribute significantly in the control of H1N1.

\section{PO-0256 ENTEROVIRUS MENINGITIS: A CASE SERIES REVIEW}

${ }^{1} \mathrm{C}$ Warren, ${ }^{2} \mathrm{P}$ Chakrabarti, ${ }^{1} \mathrm{Y}$ Kumar. ${ }^{1}$ Neonatal Unit, Royal Cornwall Hospital, Truro, UK; ${ }^{2}$ Clinical Microbiology, Royal Cornwall Hospital, Truro, UK

\subsection{6/archdischild-2014-307384.906}

Background and aims Meningitis due to Enterovirus infection is usually mild and self-limiting, particularly affecting young infants. We found that we were increasingly diagnosing the condition and undertook a retrospective review, exploring case characteristics and whether increasing use of virology detection reduced antibiotic course length, having previously rarely employed these techniques.

Methods All cases identified as being diagnosed with meningitis with a positive enterovirus polymerase chain reaction (PCR) result between January 2012 and December 2012, below 16 years of age, were retrospectively reviewed.

Results Nine cases of enterovirus meningitis were identified in the time period. Seven were infants aged 3 months or less, with two patients aged 11 and 15 years respectively. There were five 